$11-1-2011$

\title{
Explicit Equations for ACF in Autoregressive Processes In the Presence of Heteroscedasticity Disturbances
}

Samir Safi

The Islamic University of Gaza, samirsafi@gmail.com

Follow this and additional works at: http://digitalcommons.wayne.edu/jmasm

Part of the Applied Statistics Commons, Social and Behavioral Sciences Commons, and the Statistical Theory Commons

\section{Recommended Citation}

Safi, Samir (2011) "Explicit Equations for ACF in Autoregressive Processes In the Presence of Heteroscedasticity Disturbances," Journal of Modern Applied Statistical Methods: Vol. 10 : Iss. 2 , Article 20. DOI: $10.22237 /$ jmasm/1320121140

Available at: http://digitalcommons.wayne.edu/jmasm/vol10/iss2/20 


\title{
Explicit Equations for ACF in Autoregressive Processes In the Presence of Heteroscedasticity Disturbances
}

\author{
Samir Safi \\ The Islamic University of Gaza, \\ Gaza
}

The autocorrelation function, $\mathrm{ACF}$, is an important guide to the properties of a time series. Explicit equations are derived for ACF in the presence of heteroscedasticity disturbances in $p^{\text {th }}$ order autoregressive, $A R(p)$, processes. Two cases are presented: (1) when the disturbance term follows the general covariance matrix, $\Sigma$, and (2) when the diagonal elements of $\Sigma$ are not all identical but $\sigma_{\mathrm{i}, \mathrm{j}}=0 \forall \mathrm{i} \neq \mathrm{j}$.

Key words: Heteroscedasticity, homoscedasticity, autocorrelation, autoregressive, covariance, disturbance, time series.

\section{Introduction}

When disturbance terms are identically distributed, it implies that they have the same variance for all observations: this is known as homoscedasticity. If they are not, it causes serious problems for estimates and must be corrected in order to obtain reliable estimates. A sequence, or a vector, of random variables is heteroscedastic if the random variables have different variances. Heteroscedastic means differing variance and is derived from the Greek hetero, meaning different, and skedasis, meaning dispersion. The word heteroscedasticity indicates a time-varying variance and is a deviation from the identically distributed assumption because the variances are not the same for each value.

Heteroscedasticity occurs when observations are based on average data and in a number of random coefficient models. It has two forms, conditional and unconditional. Conditional heteroscedasticity identifies nonconstant volatility when future periods of high and low volatility cannot be identified.

Unconditional heteroscedasticity is when future periods of high and low volatility

Samir Safi is an Associate Professor of Statistics. Email: samirsafi@gmail.com. can be identified. For example, periods of low and high volatility for the prices of stocks and bonds cannot be predicted over any period of time, and therefore would be described as conditional heteroscedasticity. By contrast, unconditional heteroscedasticity can be used discussing variables that have identifiable seasonal variability, such as electricity usage.

The consequences of heteroscedasticity are problematic in general, and it is well known that the consequences of heteroscedasticity for ordinary least squares (OLS) estimation are very serious. Although parameter estimates remain unbiased, they are no longer efficient, meaning they are no longer best linear unbiased estimators (BLUE) among the class of all the linear unbiased estimators. The standard errors typically computed for the least squares estimators are no longer appropriate, hence, confidence intervals and hypothesis tests that use these standard errors are invalid. Because the estimated error's variance-covariance is not efficient, it invalidates the t-statistic, sometimes making insignificant variables appear to be statistically significant. Heteroscedasticity causes the OLS estimates of the standard error to be biased, leading to unreliable hypothesis testing. The most serious implication of heteroscedasticity is a misleading inference when the standard tests are used such as $t$ and $F$ tests. 


\section{AUTOREGRESSIVE PROCESSES IN THE PRESENCE OF HETEROSCEDASTICITY}

The disturbance term in time series data is modeled under an assumption of constant variance and the assumption of heteroscedastic disturbances has traditionally been considered in the context of cross-sectional data. With time series data the disturbance term is modeled with some kind of stochastic process, and most of the conventional stochastic processes assume homoscedasticity (Judge, et al., 1985). The econometrician Robert Engle won the 2003 Nobel Memorial Prize for Economics for his studies on regression analysis in the presence of heteroscedasticity, which led to his formulation of the AutoRegressive Conditional Heteroscedasticity (ARCH) modeling technique.

\section{Background}

Heteroscedasticity is a problem often faced by statisticians and econometricians. A wealth of literature related to estimating and testing heteroscedasticity exists, see for example, Wallentin and Agren (2002), Kalirajan (1989), Evans and King (1988) and Farebrother (1987).

Safi (2009) derived explicit equations for ACF in the presence of heteroscedasticity disturbances in first-order autoregressive, $A R(1)$, process. He showed two cases: (1) when the disturbance follows the general covariance matrix, $\Sigma$, and (2) when the diagonal elements of $\Sigma$ are not all identical but $\sigma_{\mathrm{i}, \mathrm{j}}=0 \forall \mathrm{i} \neq \mathrm{j}$, that is, $\Sigma=\operatorname{diag}\left(\sigma_{11}, \sigma_{22}, \ldots, \sigma_{\mathrm{tt}}\right)$. This article extends the Safi (2009) results for the general autoregressive, $A R(p)$, process.

Praetz (2008) discussed the effect of auto-correlated disturbances when they are not modeled on statistics used in drawing inferences in the multiple linear regression model. $\mathrm{He}$ derived biases for the $\mathrm{F}$ and $\mathrm{R}^{2}$ statistics and evaluated them numerically. He discussed the reflections for empirical research on the causes, detection and treatment of autocorrelation.

Bera, et al. (2005) investigated conditional and unconditional heteroscedasticities as well as normality in the market model. They showed that conditional heteroscedasticity is more widespread than unconditional heteroscedasticity, suggesting the necessity of model refinements that take conditional heteroscedasticity into account. They provided an alternative estimation of betas of individual securities and portfolios based on the autoregressive conditional heteroscedastic (ARCH) model introduced by Engle. The efficiency of the market model coefficients is markedly improved across all firms in the sample through the ARCH technique. Demos (2000) derived expressions for the autocovariance of the observed series and the squared errors as a function of the parameters, something which facilitates the comparison of the observed properties of the data with the theoretical properties of the models, and consequently may play an important part in model identification.

Studies of many econometric time series models for financial markets reveal that it is unreasonable to assume that conditional variance of the disturbance term is constant as it for many stochastic processes. Two exceptions are the heteroscedastic stochastic processes proposed by Engle (1982) and Cragg (1982). Engle (1982), showed that, for many economic models, it is unreasonable to assume that the conditional forecast variance $\operatorname{var}\left(\mathrm{y}_{\mathrm{t}} \mid \mathrm{y}_{\mathrm{t}-1}\right)$ is constant, and that is more realistic to assume that $\operatorname{var}\left(\mathrm{y}_{\mathrm{t}} \mid \mathrm{y}_{\mathrm{t}-1}\right)$ depends on $\mathrm{y}_{\mathrm{t}-1}$.

Bumb, and Kelejian (1983) studied the auto-correlated and heteroscedastic disturbances in linear regression analysis. They discussed various procedures to test for the possibility that the disturbance terms of a linear regression model are auto-correlated in a first order process with a constant autoregressive coefficient.

\section{Autocorrelation Function (ACF)}

The autocorrelation function (ACF), is an important guide to the properties of a time series. It measures the correlation between observations at different distances apart. This behavior is a powerful tool to identify a preliminary time series model. The ACF provides a better understanding of correlation structure of the data and, within the Box Jenkins framework, a rough idea of the order of the components to be used in any autoregressive model. The estimate of ACF may suggest which of the many possible stationary time series models is a suitable candidate for representing 
the dependence in the data, Brockwell and Davis (2002). The forms of the explicit equations depend on the autoregressive coefficients.

\section{General Heteroscedastic Autocorrelation} Function (GHACF)

Autoregressive processes are regressions on themselves. In other words, in autoregressive processes, the current value of the process $Z_{t}$ is expressed as a finite linear combination of the $p$ most recent past values of itself plus an innovation term $e_{t}$ which incorporates everything new in the series at time $t$ that is not explained by past values. Thus, for every $t$, it is assumed that $e_{t}$ is independent of $Z_{t-1}, Z_{t-2}, \ldots$ If the values of a process at equally spaced times $t, t-1, t-2, \ldots$, denoted by $\mathrm{Z}_{\mathrm{t}}, \mathrm{Z}_{\mathrm{t}-1}, \mathrm{Z}_{\mathrm{t}-2}, \ldots$, then $\mathrm{Z}_{\mathrm{t}}=\phi_{1} \mathrm{Z}_{\mathrm{t}-1}+\phi_{2} \mathrm{Z}_{\mathrm{t}-2}+\cdots+\phi_{\mathrm{p}} \mathrm{Z}_{\mathrm{t}-\mathrm{p}}+\mathrm{e}_{\mathrm{t}}$ is called a $p^{\text {th }}$ order autoregressive process, abbreviated $A R(p)$.

The $p^{\text {th }}$ order autoregressive model may be written in terms of backward shift operator B as:

$$
\left(1-\phi_{1} \mathrm{~B}-\cdots-\phi_{\mathrm{p}} \mathrm{B}^{\mathrm{p}}\right) \mathrm{Z}_{\mathrm{t}}=\phi(\mathrm{B}) \mathrm{Z}_{\mathrm{t}}=\mathrm{e}_{\mathrm{t}} .
$$

A special notation used to simplify the representation of lag values, with $B^{j} Z_{t}=Z_{t-j}$. $Z_{t}$ is the time series under investigation and $e_{t}$ is the white noise series normally distributed with mean zero and variance $\sigma_{\mathrm{e}}^{2}$. For the general $A R(p)$ process, $\mathrm{Z}_{\mathrm{t}}=\phi^{-1}(\mathrm{~B}) \mathrm{e}_{\mathrm{t}}$, results in

$$
\phi(B)=\left(1-G_{1} B\right)\left(1-G_{2} B\right) \cdots\left(1-G_{p} B\right)
$$

where $\mathrm{G}_{1}^{-1}, \ldots, \mathrm{G}_{\mathrm{p}}^{-1}$ are the roots of $\phi(\mathrm{B})=0$, and expanding $\phi^{-1}(\mathrm{~B})$ in partial fractions yields

$$
Z_{t}=\phi^{-1}(B) e_{t}=\sum_{i=1}^{p} \frac{K_{i}}{1-G_{i} B} e_{t} .
$$

(See for example Box, et al., 1994.) Thus, if $\psi(B)=\phi^{-1}(B)$ is to be a convergent series for $|\mathrm{B}| \leq 1$, then the weights $\psi_{\mathrm{j}}=\sum_{\mathrm{i}=1}^{\mathrm{p}} \mathrm{K}_{\mathrm{i}} \mathrm{G}_{\mathrm{i}}^{\mathrm{j}}$ must be absolutely summable so that the $A R(p)$ will represent a stationary process, $\left|\mathrm{G}_{\mathrm{i}}\right|<1$ for $i=1,2, \ldots, p$. Equivalently, the roots of $\phi(\mathrm{B})=0$ must lie outside the unit circle. From the relation $\phi(\mathrm{B}) \psi(\mathrm{B})=1$ it follows that the weights $\psi_{\mathrm{j}}$ for the $A R(p)$ process satisfy the difference equation:

$$
\psi_{\mathrm{j}}=\phi_{1} \psi_{\mathrm{j}-1}+\phi_{2} \psi_{\mathrm{j}-2}+\cdots+\phi_{\mathrm{p}} \psi_{\mathrm{j}-\mathrm{p}}, \mathrm{j}>0
$$

with $\psi_{0}=1$ and $\psi_{\mathrm{j}}=0$ for $\mathrm{j}<0$, from which the weights $\psi_{\mathrm{j}}$ can easily be computed recursively in terms of the $\phi_{\mathrm{i}}$.

The $A R(p)$ autoregressive process $\mathrm{Z}_{\mathrm{t}}=\phi^{-1}(\mathrm{~B}) \mathrm{e}_{\mathrm{t}}$ may be written as:

$$
Z_{t}=\sum_{j=0}^{\infty} \psi_{j} e_{t-j}, \quad t=0, \pm 1, \pm 2, \ldots
$$

It is assumed that the disturbance term has mean zero, $\mathrm{E}(\mathbf{e})=\mathbf{0}$, and the covariance matrix $\operatorname{Cov}\left(\mathrm{e}_{\mathrm{i}}, \mathrm{e}_{\mathrm{j}}\right)=\Sigma$ where:

$$
\Sigma=\left[\begin{array}{cccc}
\sigma_{11} & \sigma_{12} & \ldots & \sigma_{1 \mathrm{t}} \\
\sigma_{21} & \sigma_{22} & \ldots & \sigma_{2 \mathrm{t}} \\
\vdots & \vdots & \ddots & \vdots \\
\sigma_{\mathrm{t} 1} & \sigma_{\mathrm{t} 2} & \ldots & \sigma_{\mathrm{tt}}
\end{array}\right]
$$

Definition 1

The covariance between $Z_{t}$ and $Z_{t+k}$, separated by $\mathrm{k}$ intervals of time (which under the stationary assumption must be the same for all $t$ ) is called the autocovariance function at lag $\mathrm{k}$ (ACVF) and is defined by 


\section{AUTOREGRESSIVE PROCESSES IN THE PRESENCE OF HETEROSCEDASTICITY}

$$
\gamma_{k}=\operatorname{Cov}\left(Z_{t}, Z_{t+k}\right)=E\left[\left(Z_{t}-\mu\right)\left(Z_{t+k}-\mu\right)\right],
$$

assuming that $Z_{t}$ has zero mean. A nonzero mean can be introduced by replacing $Z_{t}$ by $\mathrm{Z}_{\mathrm{t}-\mu}$ throughout the equations.

Definition 2

The autocorrelation function at lag $\mathrm{k}$, that is the correlation between $Z_{t}$ and $Z_{t+k}$, is defined by

$$
\rho_{\mathrm{k}}=\frac{\gamma_{\mathrm{k}}}{\gamma_{0}}
$$

where $\gamma_{0}=\sigma_{\mathrm{Z}}^{2}$ is the same at time $\mathrm{t}+\mathrm{k}$ as at time t.

\section{Lemma 1}

Consider the general $A R(p)$ process, $\mathrm{Z}_{\mathrm{t}}=\sum_{\mathrm{j}=0}^{\infty} \psi_{\mathrm{j}} \mathrm{e}_{\mathrm{t}-\mathrm{j}}$, with $\mathrm{E} \quad\left(\mathbf{e}_{\mathrm{t}}\right)=\mathbf{0}$, and $\operatorname{Cov}\left(\mathrm{e}_{\mathrm{i}}, \mathrm{e}_{\mathrm{j}}\right)=\Sigma$, where $\Sigma$ is given in (4). The autocovariance function at lag $\mathrm{k}$ is given by

$$
\gamma_{k}=\sum_{j=0}^{t-k-1} \sum_{i=0}^{t-1} \psi_{i} \psi_{j} \sigma_{t-i, t-k-j} .
$$

Proof

$$
\text { Using (3), }
$$

$$
\begin{aligned}
\mathrm{Z}_{\mathrm{t}} \mathrm{Z}_{\mathrm{t}-\mathrm{k}} & =\left(\sum_{\mathrm{i}=0}^{\mathrm{t}-1} \psi_{\mathrm{i}} \mathrm{e}_{\mathrm{t}-\mathrm{i}}\right)\left(\sum_{\mathrm{j}=0}^{\mathrm{t}-\mathrm{k}-1} \psi_{\mathrm{j}} \mathrm{e}_{\mathrm{t}-\mathrm{k}-\mathrm{j}}\right) \\
& =\sum_{\mathrm{j}=0}^{\mathrm{t}-\mathrm{k}-1} \sum_{\mathrm{i}=0}^{\mathrm{t}-1} \psi_{\mathrm{i}} \psi_{\mathrm{j}} \mathrm{e}_{\mathrm{t}-\mathrm{i}} \mathrm{e}_{\mathrm{t}-\mathrm{k}-\mathrm{j}} .
\end{aligned}
$$

and using (5), the ACVF at lag $\mathrm{k}$ is

$$
\begin{aligned}
\gamma_{k} & =E\left(Z_{t} Z_{t-k}\right) \\
& =E\left[\sum_{j=0}^{t-k-1} \sum_{i=0}^{t-1} \psi_{i} \psi_{j} e_{t-i} e_{t-k-j}\right] \\
& =\sum_{j=0}^{t-k-1} \sum_{i=0}^{t-1} \psi_{i} \psi_{j} E\left(e_{t-i} e_{t-k-j}\right) \\
& =\sum_{j=0}^{t-k-1} \sum_{i=0}^{t-1} \psi_{i} \psi_{j} \sigma_{t-i} \sigma_{t-k-j} .
\end{aligned}
$$

Theorem 1: Deriving the GHACF at Lag $\mathrm{k}$ when $\sigma_{\mathrm{i}, \mathrm{j}} \neq 0$ for all $\mathrm{i} \neq \mathrm{j}$ In an $A R(p)$ Process

Consider the general $\mathrm{AR}(\mathrm{p})$ process $Z_{t}=\sum_{j=0}^{\infty} \psi_{j} e_{t-j}, \psi_{j}$ is given in (2), with $E\left(\mathbf{e}_{t}\right)=$ 0, and $\operatorname{Cov}\left(\mathrm{e}_{\mathrm{i}}, \mathrm{e}_{\mathrm{j}}\right)=\Sigma$, where $\Sigma$ is given in (4), with $\sigma_{i, j} \neq 0 \forall i \neq j$, then the GHACF at lag $\mathrm{k}$ is given by:

$$
\rho_{\mathrm{k}}=\frac{\sum_{\mathrm{j}=0}^{\mathrm{t}-\mathrm{k}-1} \sum_{\mathrm{i}=0}^{\mathrm{t}-1} \psi_{\mathrm{j}} \psi_{\mathrm{i}} \sigma_{\mathrm{t}-\mathrm{i}, \mathrm{t}-\mathrm{j}-\mathrm{k}}}{\sum_{\mathrm{j}=0}^{\mathrm{t}-1} \sum_{\mathrm{i}=0}^{\mathrm{t}-1} \psi_{\mathrm{j}} \psi_{\mathrm{i}} \sigma_{\mathrm{t}-\mathrm{j}, \mathrm{t}-\mathrm{i}}} .
$$

Proof

Using (7), the ACVF at lag 0 is

$\gamma_{0}=$

$\psi_{0}^{2} \sigma_{\mathrm{t}, \mathrm{t}}+\psi_{1} \sigma_{\mathrm{t}, \mathrm{t}-1}+\psi_{2} \sigma_{\mathrm{t}, \mathrm{t}-2}+\psi_{3} \sigma_{\mathrm{t}, \mathrm{t}-3}+\cdots+\psi_{\mathrm{t}-1} \sigma_{\mathrm{t}, 1}+$ $\psi_{1} \sigma_{\mathrm{t}-1, \mathrm{t}}+\psi_{1}^{2} \sigma_{\mathrm{t}-1, \mathrm{t}-1}+\psi_{1} \psi_{2} \sigma_{\mathrm{t}-1, \mathrm{t}-2}+\cdots+\psi_{1} \psi_{\mathrm{t}-1} \sigma_{\mathrm{t}-1,1}+$ $\psi_{2} \sigma_{\mathrm{t}-2, \mathrm{t}}+\psi_{2} \psi_{1} \sigma_{\mathrm{t}-2, \mathrm{t}-1}+\psi_{2}^{2} \sigma_{\mathrm{t}-2, \mathrm{t}-2}+\cdots+\psi_{2} \psi_{\mathrm{t}-1} \sigma_{\mathrm{t}-2,1}+$ $\psi_{\mathrm{t}-1} \sigma_{1, \mathrm{t}}+\psi_{\mathrm{t}-1} \psi_{1} \sigma_{1, \mathrm{t}-1}+\psi_{\mathrm{t}-1} \psi_{2} \sigma_{1, \mathrm{t}-2}+\cdots+\psi_{\mathrm{t}-1} \psi_{\mathrm{t}-2} \sigma_{1,2}+\psi_{\mathrm{t}-1}^{2} \sigma_{1,1}$.

Collecting terms, the ACVF at lag 0, that is, the variance of the process is:

$$
\gamma_{0}=\sum_{j=0}^{t-1} \sum_{i=0}^{t-1} \psi_{j} \psi_{i} \sigma_{t-j, t-i} .
$$


Using (7), the ACVF at lag 1 is

$\gamma_{1}=$

$\sigma_{\mathrm{t}, \mathrm{t}-1}+\psi_{1} \sigma_{\mathrm{t}, \mathrm{t}-2}+\psi_{2} \sigma_{\mathrm{t}, \mathrm{t}-3}+\psi_{3} \sigma_{\mathrm{t}, \mathrm{t}-4}+\cdots+\psi_{\mathrm{t}-2} \sigma_{\mathrm{t}, 1}+$

$\psi_{1} \sigma_{\mathrm{t}-1, \mathrm{t}-1}+\psi_{1}^{2} \sigma_{\mathrm{t}-1, \mathrm{t}-2}+\psi_{1} \psi_{2} \sigma_{\mathrm{t}-1, \mathrm{t}-3}+\cdots+\psi_{1} \psi_{\mathrm{t}-2} \sigma_{\mathrm{t}-1,1}+$

$\psi_{2} \sigma_{\mathrm{t}-2, \mathrm{t}-1}+\psi_{2} \psi_{1} \sigma_{\mathrm{t}-2, \mathrm{t}-2}+\psi_{2}^{2} \sigma_{\mathrm{t}-2, \mathrm{t}-3}+\cdots+\psi_{2} \psi_{\mathrm{t}-2} \sigma_{\mathrm{t}-2,1}+$

$\psi_{\mathrm{t}-1} \sigma_{1, \mathrm{t}-1}+\psi_{\mathrm{t}-1} \psi_{1} \sigma_{1, \mathrm{t}-2}+\psi_{\mathrm{t}-1} \psi_{2} \sigma_{1, \mathrm{t}-3}+\cdots+\psi_{\mathrm{t}-1} \psi_{\mathrm{t}-2} \sigma_{1,1}$.

Collecting terms, the ACVF at lag 1 is

$$
\gamma_{1}=\sum_{\mathrm{j}=0}^{\mathrm{t}-2} \sum_{\mathrm{i}=0}^{\mathrm{t}-1} \psi_{\mathrm{j}} \psi_{\mathrm{i}} \sigma_{\mathrm{t}-\mathrm{i}, \mathrm{t}-\mathrm{j}-1}
$$

similarly, the ACVF at lag $\mathrm{k}$ is

$$
\gamma_{\mathrm{k}}=\sum_{\mathrm{j}=0}^{\mathrm{t}-\mathrm{k}-1} \sum_{\mathrm{i}=0}^{\mathrm{t}-1} \psi_{\mathrm{j}} \psi_{\mathrm{i}} \sigma_{\mathrm{t}-\mathrm{i}, \mathrm{t}-\mathrm{j}-\mathrm{k}}
$$

Dividing (13) by (10), results in (8), which completes the proof.

Corollary 1: GHACF at Lag $\mathrm{k}$ for an $A R(1)$ Process

Consider an $A R(1)$ process $\mathrm{Z}_{\mathrm{t}}=\sum_{\mathrm{j}=0}^{\infty} \psi_{\mathrm{j}} \mathrm{e}_{\mathrm{t}-\mathrm{j}}, \psi_{\mathrm{j}}=\phi \psi_{\mathrm{j}-1}$ with $\mathrm{E}\left(\mathbf{e}_{\mathrm{t}}\right)=\mathbf{0}$, and $\operatorname{Cov}\left(\mathrm{e}_{\mathrm{i}}, \mathrm{e}_{\mathrm{j}}\right)=\Sigma$, where $\Sigma$ is given in (4), with $\sigma_{\mathrm{i}, \mathrm{j}} \neq 0 \forall \mathrm{i} \neq \mathrm{j}$. The GHACF at lag $\mathrm{k}$ is given by

$$
\rho_{k}=\frac{\sum_{j=0}^{t-k-1} \sum_{i=0}^{t-1} \phi^{j+i} \sigma_{t-i, t-j-k}}{\sum_{j=0}^{t-1} \sum_{i=0}^{t-1} \phi^{j+i} \sigma_{t-j, t-i}} .
$$

Proof

For an $A R(1)$ process, because $\psi_{\mathrm{j}}=\phi \psi_{\mathrm{j}-1}$, it follows that $\psi_{\mathrm{j}}=\phi^{\mathrm{j}}$, for $\mathrm{j} \geq 0$. From equations (10) and (13),

$$
\gamma_{0}=\sum_{j=0}^{\mathrm{t}-1} \sum_{\mathrm{i}=0}^{\mathrm{t}-1} \phi^{\mathrm{j}+\mathrm{i}} \sigma_{\mathrm{t}-\mathrm{j}, \mathrm{t}-\mathrm{i}}
$$

and

$$
\gamma_{k}=\sum_{j=0}^{t-k-1} \sum_{i=0}^{t-1} \phi^{j+i} \sigma_{t-i, t-j-k}
$$

are obtained, thus completing the proof.

Heteroscedastic Autocorrelation Function (HACF)

Heteroscedasticity exists if the diagonal elements of $\Sigma$ in (4) are not all identical and the disturbance term is free from autocorrelation, meaning, the disturbances are pairwise uncorrelated. This assumption is likely to be realistic one when using cross-sectional data. In this case $\Sigma$ can be written as a diagonal matrix with the $i^{\text {th }}$ diagonal element given by $\sigma_{\mathrm{ii}}$. Assume $E\left(\mathbf{e}_{\mathbf{t}}\right)=\mathbf{0}$, and $\operatorname{Cov}\left(\mathrm{e}_{\mathrm{i}}, \mathrm{e}_{\mathrm{j}}\right)=\Sigma$, where $\Sigma=\operatorname{diag}\left(\sigma_{11}, \sigma_{22}, \ldots, \sigma_{\mathrm{tt}}\right)$. Thus,

$$
\Sigma=\left[\begin{array}{cccc}
\sigma_{11} & 0 & \ldots & 0 \\
0 & \sigma_{22} & \ldots & 0 \\
\vdots & \vdots & \ddots & \vdots \\
0 & 0 & \ldots & \sigma_{\mathrm{tt}}
\end{array}\right]
$$

Theorem 2: HACF, at Lag $\mathrm{k}$ when $\sigma_{\mathrm{i}, \mathrm{j}}=0$ for all $\mathrm{i} \neq \mathrm{j}$, i.e. $\Sigma=\operatorname{diag}\left(\sigma_{11}, \sigma_{22}, \ldots, \sigma_{\mathrm{tt}}\right)$ In an $A R(p)$ Process

Consider the general $A R(p)$ process $\mathrm{Z}_{\mathrm{t}}=\sum_{\mathrm{j}=0}^{\infty} \psi_{\mathrm{j}} \mathrm{e}_{\mathrm{t}-\mathrm{j}}, \psi_{\mathrm{j}}$ as given in (2), with $\mathrm{E}\left(\mathbf{e}_{\mathrm{t}}\right)=$ 0 and $\operatorname{Cov}\left(\mathrm{e}_{\mathrm{i}}, \mathrm{e}_{\mathrm{j}}\right)=\Sigma$, with $\sigma_{\mathrm{i}, \mathrm{j}}=0 \forall \mathrm{i} \neq \mathrm{j}$, that is, $\Sigma=\operatorname{diag}\left(\sigma_{11}, \sigma_{22}, \ldots, \sigma_{\mathrm{tt}}\right)$ as given in (15). The HACF at lag $\mathrm{k}$ is then given by

$$
\rho_{\mathrm{k}}=\frac{\sum_{\mathrm{i}=\mathrm{k}}^{\mathrm{t}-1} \psi_{\mathrm{i}} \psi_{\mathrm{i}-\mathrm{k}} \sigma_{\mathrm{t}-\mathrm{i}, \mathrm{t}-\mathrm{i}}}{\sum_{\mathrm{i}=0}^{\mathrm{t}-1} \psi_{\mathrm{i}}^{2} \sigma_{\mathrm{t}-\mathrm{i}, \mathrm{t}-\mathrm{i}}} .
$$




\section{AUTOREGRESSIVE PROCESSES IN THE PRESENCE OF HETEROSCEDASTICITY}

Proof

Using (9) with $\sigma_{i, j}=0 \forall i \neq j$, the

$\mathrm{ACVF}$ at lag 0 is

$\gamma_{0}=$

$\psi_{0}^{2} \sigma_{\mathrm{t}, \mathrm{t}}+\psi_{1}^{2} \sigma_{\mathrm{t}-1, \mathrm{t}-1}+\psi_{2}^{2} \sigma_{\mathrm{t}-2, \mathrm{t}-2}+\psi_{3}^{2} \sigma_{\mathrm{t}-3, \mathrm{t}-3}+\cdots+\psi_{\mathrm{t}-1}^{2} \sigma_{1,1}$

and the ACVF at lag 0 , that is, the variance of the general $A R(p)$ process is

$$
\gamma_{0}=\sum_{\mathrm{i}=0}^{\mathrm{t}-1} \psi_{\mathrm{i}}^{2} \sigma_{\mathrm{t}-\mathrm{i}, \mathrm{t}-\mathrm{i}}
$$

Using (11) with $\sigma_{i, j}=0 \forall i \neq j$, the ACVF at lag 1 is

$$
\begin{aligned}
& \gamma_{1}= \\
& \psi_{1} \sigma_{\mathrm{t}-1, \mathrm{t}-1}+\psi_{2} \psi_{1} \sigma_{\mathrm{t}-2, \mathrm{t}-2}+\cdots+\psi_{\mathrm{t}-1} \psi_{\mathrm{t}-2} \sigma_{1,1}
\end{aligned}
$$

so that the ACVF at lag 1 is

$$
\gamma_{1}=\sum_{\mathrm{i}=1}^{\mathrm{t}-1} \psi_{\mathrm{i}} \psi_{\mathrm{i}-1} \sigma_{\mathrm{t}-\mathrm{i}, \mathrm{t}-\mathrm{i}}
$$

Similarly, the ACVF at lag $\mathrm{k}$ is

$$
\gamma_{\mathrm{k}}=\sum_{\mathrm{i}=\mathrm{k}}^{\mathrm{t}-1} \psi_{\mathrm{i}} \psi_{\mathrm{i}-\mathrm{k}} \sigma_{\mathrm{t}-\mathrm{i}, \mathrm{t}-\mathrm{i}}
$$

Dividing (19) by (17), results in (16), which completes the proof.

Corollary 2: $\mathrm{HACF}$ at Lag $\mathrm{k}$ for an $A R(1)$ Process Consider an $A R(1)$ process, $\mathrm{Z}_{\mathrm{t}}=\sum_{\mathrm{j}=0}^{\infty} \psi_{\mathrm{j}} \mathrm{e}_{\mathrm{t}-\mathrm{j}}, \psi_{\mathrm{j}}=\phi \psi_{\mathrm{j}-1}$, with $\mathrm{E}\left(\mathbf{e}_{\mathrm{t}}\right)=\mathbf{0}$, and $\operatorname{Cov}\left(\mathrm{e}_{\mathrm{i}}, \mathrm{e}_{\mathrm{j}}\right)=\Sigma$, with $\sigma_{\mathrm{i}, \mathrm{j}}=0 \forall \mathrm{i} \neq \mathrm{j}$, that is, $\Sigma=\operatorname{diag}\left(\sigma_{11}, \sigma_{22}, \ldots, \sigma_{\mathrm{tt}}\right)$ as given in (15). Then the HACF at lag $\mathrm{k}$ is given by

$$
\rho_{k}=\frac{\sum_{\mathrm{i}=\mathrm{k}}^{\mathrm{t}-1} \phi^{2 \mathrm{i}-\mathrm{k}} \sigma_{\mathrm{t}-\mathrm{i}, \mathrm{t}-\mathrm{i}}}{\sum_{\mathrm{i}=0}^{\mathrm{t}-1} \phi^{2 \mathrm{i}} \sigma_{\mathrm{t}-\mathrm{i}, \mathrm{t}-\mathrm{i}}} .
$$

Proof

For an $A R(1)$ process, because $\psi_{\mathrm{j}}=\phi \psi_{\mathrm{j}-1}$, it follows that $\psi_{\mathrm{j}}=\phi^{\mathrm{j}}$, for $\mathrm{j} \geq 0$. From equations (17) and (19), $\gamma_{0}=\sum_{\mathrm{i}=0}^{\mathrm{t}-1} \phi^{2 \mathrm{i}} \sigma_{\mathrm{t}-\mathrm{i}, \mathrm{t}-\mathrm{i}}$ and $\gamma_{\mathrm{k}}=\sum_{\mathrm{i}=\mathrm{k}}^{\mathrm{t}-1} \phi^{2 \mathrm{i}-\mathrm{k}} \sigma_{\mathrm{t}-\mathrm{i}, \mathrm{t}-\mathrm{i}}$ are obtained and the proof is complete.

Special Case

Homoscedasticity exists if the diagonal elements of $\Sigma$ in (4) are all identical and the disturbance term, e, is free from autocorrelation, that is, $\sigma_{\mathrm{ij}}=0 \forall \mathrm{i} \neq \mathrm{j}$. In this case, the disturbance term is a sequence of independent, identically distributed random variables.

Corollary 3.3: $\mathrm{ACF}$ at $\mathrm{Lag} \mathrm{k}$ for an $A R(1)$ Process Using Theorem (3.2)

Consider an $A R(1)$ process, $Z_{t}=\sum_{j=0}^{\infty} \psi_{j} e_{t-j}, \quad \psi_{j}=\phi \psi_{j-1}$, with $E\left(e_{t}\right)=\mathbf{0}$, $\sigma_{\mathrm{i}, \mathrm{j}}=0 \forall \mathrm{i} \neq \mathrm{j}$, and $\operatorname{Var}\left(\mathrm{e}_{\mathrm{t}}\right)=\sigma^{2} \forall \mathrm{t}$. For an $A R(1), \quad \psi_{\mathrm{j}}=\phi^{\mathrm{j}}$ for $\mathrm{j} \geq 0$, taking $\mathrm{t} \rightarrow \infty$ in equations (17) through (19), results in

$$
\gamma_{0}=\sigma^{2} \sum_{i=0}^{\infty} \phi^{2 i}=\frac{\sigma^{2}}{1-\phi^{2}}
$$

$$
\gamma_{1}=\sigma^{2} \sum_{i=1}^{\infty} \phi^{2 i-1}=\phi \frac{\sigma^{2}}{1-\phi^{2}}
$$

and

$$
\gamma_{\mathrm{k}}=\sigma^{2} \sum_{\mathrm{i}=\mathrm{k}}^{\infty} \phi^{2 \mathrm{i}-\mathrm{k}}=\phi^{\mathrm{k}} \frac{\sigma^{2}}{1-\phi^{2}},
$$

respectively. The ACF at lag $\mathrm{k}$ is then given by $\rho_{\mathrm{k}}=\phi^{\mathrm{k}}, \mathrm{k} \geq 0$, which is the well-known ACF for an $A R(1)$ process. 


\section{Conclusion}

This study investigated an important statistical problem concerning the autocorrelation function (ACF) in the presence of heteroscedasticity disturbances in $p^{\text {th }}$ order autoregressive $(A R(p))$ processes. Explicit equations were derived for ACF when the disturbance follows the general covariance matrix, $\Sigma$, and when the diagonal elements of $\Sigma$ are not all identical but $\sigma_{\mathrm{ij}}=0 \forall \mathrm{i} \neq \mathrm{j}$, i.e., $\Sigma=\operatorname{diag}\left(\sigma_{11}, \sigma_{22}, \ldots, \sigma_{\mathrm{tt}}\right)$.

Future research is needed to extend the explicit equations derived in this article for ACF in the presence of heteroscedasticity disturbances in the general form of the moving average models with order $q, M A(q)$.

\section{References}

Bera, A., Bubnys, E., \& Park, H. (2005). Conditional heteroscedasticity in the market model and efficient estimates of betas. Financial Review, 23(2), 201-214.

Box, G. E., Jenkins, G. M., \& Reinsel, G. C. (1994). Time series analysis ( ${ }^{\text {rd }}$ Ed.). New Jersey: Prentice Hall.

Brockwell, P. J., \& Davis, R. A. (2002). Introduction to time series and forecasting. New York: Springer.

Bumb, B., \& Kelejian, H. (1983). Autocorrelated and heteroscedastic disturbances in linear regression analysis: A Monte Carlo study. The Indian Journal of Statistics, 45, Series B(2), 257-270.

Cragg, J. G. (1982). Estimation and testing in time series regression models with heteroscedastic disturbances. Journal of Econometrics, 20, 135-157.
Demos, A. (2000). The autocorrelation function of conditionally heteroskedastic in mean models. Athens University of Economics and Business, Department of International and European Economic Studies.

Engle, R. F. (1982). Autoregressive Conditional Heteroscedasticity with Estimation of the variance of United Kingdom Inflation. Econometrica, 50, 987-1008.

Evans, M. A., \& King, M. L. (1988). A further class of tests for heteroscedasticity. Journal of Econometrics, 37, 265-276.

Farebrother, R. W. (1987). The statistical foundations of a class of parametric tests for heteroscedasticity. Journal of Econometrics, 36, 359-368.

Judge, G. G., Griffiths, W. E., Hill, R. C., Lütkepohl, H., \& Lee. T. (1985). The theory and practice of econometrics. New York: John Wiley and Sons.

Kalirajan, K. P. (1989). A test for heteroscedasticity and non-normality of regression residuals. Economics Letters, 30, 133-136.

Praetz, P. (2008). A note on the effect of autocorrelation on multiple regression statistics. Australian \& New Zealand Journal of Statistics, 23, 309-313.

Safi, S. (2009). Explicit equations for $\mathrm{ACF}$ in the presence of heteroscedasticity disturbances in first-order autoregressive models, AR(1). The Journal of the Islamic University of Gaza, 17(2), 97-107.

Wallentin, B., \& Agren, A. (2002). Test of heteroscedasticity in a regression model in the presence of measurement errors. Economics Letters, 76, 205-211. 